Transaminase levels followed the same trend with regard to prevalence at baseline (2.6\%) and 12 months (3.7\%). AST:ALT ratio (AAR) increased over twelve months with Pearson correlation of 0.92 .

In patients with abnormal transaminases non-invasive assessment of fibrosis (NAFLD fibrosis score) demonstrated that $20 \%$ of patients were at risk of advanced fibrosis at baseline, climbing to $80 \%$ at twelve months, without differences between groups. This is $0.5 \%$ and $3 \%$ of all participants at baseline and twelve months respectively.

Conclusion In newly diagnosed diabetes abnormalities of liver function persist in the first year of diagnosis regardless of lifestyle intervention. Estimation of fibrosis in a subgroup of patients demonstrated increased prevalence of advanced fibrosis over twelve months. This is consistent with rising AAR over the same period for the whole group. Longer-term follow up of these patients is required, but initial data are similar to cohorts described elsewhere in the literature, ${ }^{1}$ and reflect a significant risk of liver disease in patients with type 2 diabetes.

Competing interests None.

Keywords lifestyle, NALFD.

\title{
REFERENCE
}

1. Targher G, Bertolini L, Poli F, et al. Nonalcoholic fatty liver disease and risk of future cardiovascular events among type 2 diabetic patients. Diabetes 2005;54(12):3541.

\section{OC-117 PREVALENCE OF ABNORMAL LIVER FUNCTION AND RESPONSE TO LIFESTYLE INTERVENTIONS IN NEWLY DIAGNOSED TYPE 2 DIABETES: PRELIMINARY RESULTS OF A UK RANDOMISED CONTROLLED STUDY}

doi:10.1136/gut.2011.239301.117

R Parker, ${ }^{1 *}$ N Guthrie, ${ }^{2}$ N Patel, ${ }^{2}$ W Woltersdorf, ${ }^{2}$ R Andrews, ${ }^{3}$ C Dayan, ${ }^{4}$ C A McCune, ${ }^{5}$ Early ACTID ${ }^{1}$ Department of Gastroenterology, Sandwell and West Birmingham Hospitals, Birmingham, UK; ${ }^{2}$ Department of Biochemistry, University Hospitals of Bristol NHS Foundation Trust, Bristol, UK; ${ }^{3}$ Department of Diabetes and Endocrinology, Taunton and Somerset Hospitals NHS Trust, Taunton, UK; ${ }^{4}$ Department of Diabetes and Metabolism, University of Cardiff, Cardiff, UK; ${ }^{5}$ Liver Medicine, University Hospitals of Bristol NHS Foundation Trust, Bristol, UK

Introduction Non-alcoholic fatty liver disease (NAFLD) is increasingly common with a prevalence of between $20 \%$ and $34 \%$ in Europe and the US. There is a close association between NAFLD and other features of the metabolic syndrome such as insulin resistance, hypertension and obesity. We investigated liver health in patients with diabetes, and the effect of lifestyle interventions on liver health in newly diagnosed type 2 diabetics.

Methods Patients within 5-8 months of diagnosis of diabetes were identified from four centres in the South West of England. All participants received usual care for patients with diabetes. Patients were randomised to usual care alone (UC), additional dietary advice (D) or additional dietary and exercise advice (D\&E). LFTs were measured at baseline and twelve months.

Results Median age of participants was 61 years, $36 \%$ were female. Alcohol intake was modest and did not differ significantly between groups.

Modest reductions in BMI and insulin resistance were seen in groups D and D\&E: these were not significant. There was no change in blood pressure.

At baseline 24 of 424 patients (5.6\%) had any abnormality in liver function. At twelve months this was 15 of 212 patients (7.1\%). Increased prevalence was seen in all groups except D. 